\title{
Actualización curricular del Programa de Sistemas de Información y Documentación de la Universidad de La Salle- Colombia
}

\author{
Johann Pirela Morillo* \\ Nelson Javier Pulido Daza*
}

Artículo recibido:

17 de noviembre de 2015

Artículo aceptado:

27 de septiembre de 2016

\section{RESUMEN}

Se expone el proceso de investigación que dio origen a la actualización curricular del Programa de Sistemas de Información y Documentación (PSID) de la Universidad de La Salle-Colombia, enmarcado en el Proyecto Educativo y Enfoque Formativo Lasallistas (PEUL y EFL). Utilizando la triangulación de técnicas, instrumentos y acto res como estrategia metodológica en la cual se sustentó el estudio, se concretan apuestas significativas acordes al perfil del profesional de la información mediadas por una demanda laboral, que se hacen explícitas en la actualización del currículo, fortaleciendo las líneas de la bibliotecología, la archivística y los

* Universidad de La Salle, Colombia._jepirela@unisalle.edu.co

npulido@lasalle.edu.co

INVESTIGACIÓN BIBLIOTECOLÓGICA, vol.32, núm.74, enero/marzo, 2018, México, ISSN: 2448-8321. pp. 145-169 
sistemas de información con una tendencia hacia la formación investigativa, enfocada a incluir nuevas modalidades de grado, todo ello alineado con perfiles y competencias orientadoras de una nueva malla curricular articulada con líneas de investigación institucional que harán mucho más significativa y pertinente la formación de profesionales de la información. Como conclusión se aspira a que el recorrido metodológico seguido para la actualización curricular del PSID aporte insumos para la construcción de caminos metodológicos propios sobre cuya base se aborden procesos de evaluación curricular en Bibliotecología, Archivología y Ciencias de la Información en América Latina.

Palabras clave: Evaluación Curricular; Currículo de Bibliotecología y Archivística; Formación Profesional en Bibliotecología y Archivística; Planes de Estudios; Educación Bibliotecológica y Archivística

Curricular Program Update Information and Documentation Systems of La Salle's University. Colombia Johann Pirela-Morillo, Nelson-Javier Pulido-Daza

\section{Abstract}

The research leading to the updated curriculum of the Information and Documentation Systems Program (PSID) of the Universidad de La Salle-Colombia, (part of the Educational Project and Lasallian Formative Approach (PEUL and EFL) is presented. Using triangulation of techniques, instruments and actors as the basis of the methodological approach, the study makes key observations regarding professional profile of graduates and labor market demand serve to guide the curriculum update, which fortifies the areas of librarianship, archival science, and information systems through research training, while providing for new degree modalities. These updates are all aligned with institutional lines of research, making them much more meaningful and relevant to the education of information professionals. The methodology deployed for the PSID curriculum update provides 
materials for an institution to deploy its own methodological approach that can provide the foundation for curriculum evaluation processes addressing the areas of Library Science, Archivology and Information Sciences in Latin America.

Keywords: Curriculum Evaluation; Training Librarianship; Archival Training Curriculum in Library and Archives; Systems Information; Systems Information and Documentation

\section{INTRODUCCIÓN}

A nte los acelerados cambios que plantean las sociedades de la información y del conocimiento, se requiere que los currículos para la formación de profesionales de bibliotecología y archivística se ajusten a las complejidades emergentes en los entornos globales e interactivos que surgen del seno de estas sociedades, sin perder de vista la atención a los problemas prioritarios que deben atender tales profesionales en sus contextos inmediatos de actuación. Partiendo de esta premisa, la investigación que se expone presenta el proceso de investigación realizado en el Programa de Sistemas de Información y Documentación de la Universidad de La Salle-Colombia (PSID), el cual generó como resultado la propuesta de una nueva malla curricular, articulada desde el perfil de competencias y vinculada con líneas de investigación institucional para estimular el desarrollo de un espíritu crítico y creativo en los profesionales en formación.

Los referentes teóricos utilizados para abordar el proceso de indagación del que se derivó la actualización curricular se enmarcaron en los lineamientos institucionales de la Universidad de La Salle y en tendencias proyectadas para las disciplinas bibliotecológica y archivística, que se apoyan en los procesos de análisis, diseño e implementación de sistemas de información y documentación, en principios y enfoques de gestión y el desarrollo de una formación investigativa, que hará mucho más sustantiva la preparación profesional en el área.

La triangulación de técnicas (análisis documental y de tendencias, consulta con actores involucrados en el diseño, ejecución y evaluación del currículo), de instrumentos de recolección de datos (matrices de análisis de contenido y listas de cotejo) y de actores (estudiantes de últimos semestres, egresados, empleadores, docentes e investigadores adscritos al programa) fue la estrategia metodológica definida para el desarrollo del estudio. 
Los resultados apuntaron hacia una mayor articulación del perfil profesional y la malla propuesta, logrando el fortalecimiento de las líneas curriculares de bibliotecología, archivística, sistemas de información y administración, apoyada en una tendencia sobre la formación investigativa, que considera la inclusión de nuevas modalidades de grado en sintonía con lineamientos derivados de las líneas de investigación institucional.

Dentro de las conclusiones más relevantes se destaca la posibilidad de que el recorrido metodológico seguido para la actualización curricular del PSID aporte insumos para la construcción de caminos metodológicos propios sobre cuya base se aborden procesos de evaluación curricular en bibliotecología, archivología y ciencias de la información en América Latina.

\section{Aspectos CONTEXTUALES Y CONCEPTUALES DE LA ACTUALIZACióN CURRICULAR}

El proceso de actualización curricular del Programa de Sistemas de Información y Documentación, Bibliotecología y Archivística se enmarca en las tendencias proyectadas para educación superior, definidas por expertos de la Unesco (2009) y señaladas por Inciarte, Bozo y Parra (2012) en el contexto de un modelo heurístico para impulsar la reconceptualización de la universidad en América Latina. Según las autoras, frente a las complejidades de las sociedades del conocimiento, las instituciones de educación superior deben implementar acciones que les permitan posicionarse como verdaderos motores del desarrollo, sin renunciar a su condición de ambientes de creación intelectual, que supone definir currículos flexibles, con la inclusión de ejes transversales y proyectos formativos en sintonía con el avance de la ciencia y de la tecnología.

En el marco de estos argumentos iniciales es posible ubicar el proceso que generó como resultado un nuevo diseño curricular para el programa. Además de los planteamientos señalados, se tienen también las tendencias definidas por la IFLA (2013), en las cuales se ratifican las nuevas tecnologías de la información y comunicación para la expansión (en algunos casos) y restricción (en otros) del acceso a la información, junto con las oportunidades que plantea la educación en línea, que democratizará y modificará el aprendizaje global, dando paso a nuevas y más complejas formas de interacción y comunicación didáctica. También se señala la redefinición de los límites de la privacidad y la protección de datos en el contexto de sociedades cada vez más hiperconectadas, que escucharán y empoderarán nuevas voces y grupos, aunado a las influencias de la economía global de la información que seguirá su proceso de transformación debido a la expansión de las nuevas tecnologías. 
Las ideas expresadas por la IFLA (Pirela, 2015) impactan la formación de los profesionales de la información debido a que se requerirá del impulso al empoderamiento de nuevas voces y grupos sociales, a partir de estrategias formativas desarrolladas por bibliotecólogos y archivistas, quienes desde sus espacios de acción profesional orientarán procesos de apropiación de la información de una forma mucho más crítica y significativa por parte de los usuarios, y al mismo tiempo sentarán las bases para una gestión ética y responsable del conocimiento, avanzando con ello hacia la democratización de los saberes.

Otro de los referentes que podemos considerar importante para establecer los elementos contextuales de la redimensión curricular del PSID son las líneas trazadas desde la Declaración de Lyon, que plantean posibilidades relativas a la articulación de acciones para impulsar el desarrollo a partir de los servicios de información y documentación (IFLA, 2014).

En este sentido, se apuesta por el carácter transformacional del derecho a la información como elemento de desarrollo sostenible, lo cual supone además que el acceso a las fuentes sería garantía de desarrollo de las personas, sobre todo aquellas que se encuentran en situación de exclusión, todo lo cual alude al hecho de que los profesionales de la información asuman una dimensión mediadora activa en los procesos de transmisión, organización, estructuración y comprensión de la información vital para impulsar procesos de empoderamiento social.

El currículo de bibliotecología, archivística y ciencias de la información debe responder de una forma dinámica y creativa al contexto actual, por ello exponemos los aspectos de orden conceptual que sirven de marco de referencia para la actualización del currículo en el PSID. Los referentes que permitieron ubicar la redimensión curricular del programa son el Proyecto Educativo Universitario Lasallista (PEUL) (Universidad de La Salle, 2007), el Enfoque Formativo Lasallista (EFL) (Universidad de La Salle, 2008a) y los Lineamientos para la redimensión permanente de la malla curricular (Universidad de La Salle, 2008b).

En cuanto al Proyecto Educativo Universitario Lasallista, se asumen siete horizontes de sentido en torno a los cuales se estructura la acción formativa y la gestión académica en la universidad, mediante la concepción, diseño y ejecución de sus currículos. Tales horizontes son: 1 . El pensamiento social de la Iglesia; 2. La reflexión de la universidad, la cultura, la ciencia y la tecnología; 3. La reflexión educativa lasallista; 4. El desarrollo humano integral y sustentable; 5 . La democratización del conocimiento; 6. La normatividad y las políticas públicas, y 7 . Los valores de solidaridad-fraternidad, honestidad y responsabilidad social, respeto, tolerancia, esperanza y fe, cada uno de los 
cuales deben incorporarse transversalmente en las prácticas pedagógicas y didácticas, orientadas hacia la formación de profesionales integrales y éticamente comprometidos con el desarrollo social.

El EFL (Universidad de La Salle, 2008a) pretende una educación que permita el despliegue de las potencialidades gracias a una adecuada interacción con sus grupos de referencia y con la sociedad. El enfoque entiende, entonces, la educación como un proceso orientado a facilitar que las personas logren ser responsables y cultiven su sentido crítico en conexión con la interacción grupal comunitaria y social y una inteligente contextualización política y económica. La concepción de la formación integral se asume desde esta perspectiva como un proceso de construcción de sí mismo, que por medio de la intersubjetividad se orienta a desplegar las potencialidades de sus agentes formativos (educador y educando) a través de una relación pedagógica fundada en el ejercicio responsable de la autonomía, la generación significativa de conocimiento y el compromiso decisivo con la transformación de la realidad social y cultural.

Los lineamientos curriculares para la redimensión del PSID se apoyaron en la concepción del currículo en la Universidad de La Salle, el cual se asume como un proyecto en construcción permanente, con visión sistémica que involucra la interacción del medio ambiente, la ecología, la ciencia y la sociedad. Estos preceptos confluyen en un enfoque curricular que privilegia los diálogos con pedagogías que cultiven la autonomía responsable y fomenten la autorreflexión y el autoconocimiento, integren procesos de conocimiento y prácticas interdisciplinarias y se concreten en didácticas abiertas a la participación, por lo cual se enfatiza en el trabajo colaborativo y las dinámicas grupales.

En el marco de estos argumentos, que perfilan los aspectos que deben estar presentes en los procesos de actualización curricular, se plantean los retos y desafíos presentes en un mundo en constante cambio. De este modo se han considerado la exigencia de currículos flexibles, integrales, coherentes y transversales que promuevan la movilidad interna, nacional e internacional; la homologación de títulos así como la formulación y gestión de proyectos interdisciplinares.

En tal sentido, los currículos se actualizan articulando los programas por áreas del conocimiento con ejes de problemas reales sobre la bibliotecología y la archivística, fundamentos teóricos intencionados, mediación de tecnología y praxis investigativa consciente y pertinente donde se visualice el mayor énfasis en el aprendizaje y generación de conocimiento, con precisión de dimensiones humanas integrales expresadas en competencias que exigen modificación de las estrategias de evaluación, de enseñanza-aprendizaje y modalidades de grado. 
De modo pues que en el PSID, articulado a su vez con el PEUL, se abordó desde la perspectiva disciplinar la actualización de la malla curricular, sustentada en una mayor articulación con el perfil, logrando con ello una formación integral de los futuros bibliotecólogos y archivistas con énfasis en el desarrollo de habilidades investigativas, tecnológicas y gerenciales sobre la organización, administración, preservación y uso de los sistemas de información, concebidos como soporte para el desarrollo social, económico y político del país así como mediadores en la transformación y apropiación del conocimiento.

\section{RECORRIDO METODOLÓGICO PARA LOGRAR LA ACTUALIZACIÓN CURRICULAR}

El proceso de actualización consideró los lineamientos curriculares anteriormente mencionados, sobre todo aquellos que insisten en la concepción del currículo como construcción cultural en constante evaluación y ajuste, de modo que esta noción permitió definir un recorrido metodológico de carácter mixto, el cual combinó técnicas como el análisis documental, de tendencias y la consulta con actores vinculados con las dinámicas de diseño, ejecución y evaluación del currículo: estudiantes de últimos semestres, egresados, profesores e investigadores adscritos al programa y empleadores representantes de diversas organizaciones del sector socioproductivo público y privado.

El análisis documental se aplicó a las tendencias internacionales identificadas como prioritarias, las cuales se reseñan en documentos de IFLA. Este tipo de análisis también se utilizó en el desmonte de diseños curriculares de bibliotecología, archivística y ciencia de la información vigentes en Colombia con el objeto de identificar la tendencia de la formación en el país y las áreas de mayor fortaleza en cada una de las escuelas y programas. También se recurrió a la técnica de los grupos de discusión, en los cuales participaron estudiantes de los últimos semestres del programa, egresados, docentes e investigadores adscritos. En paralelo a lo anterior, se incluyó la participación de empleadores de los profesionales de la información egresados, con la finalidad de explorar la percepción sobre su desempeño y detectar posibles vacíos en la formación, articulados con las necesidades puntuales de los diversos sectores productivos en los que se demandan los profesionales del PSID. La Figura 1 ilustra el recorrido metodológico seguido para generar los productos de la redimensión curricular del PSID. 


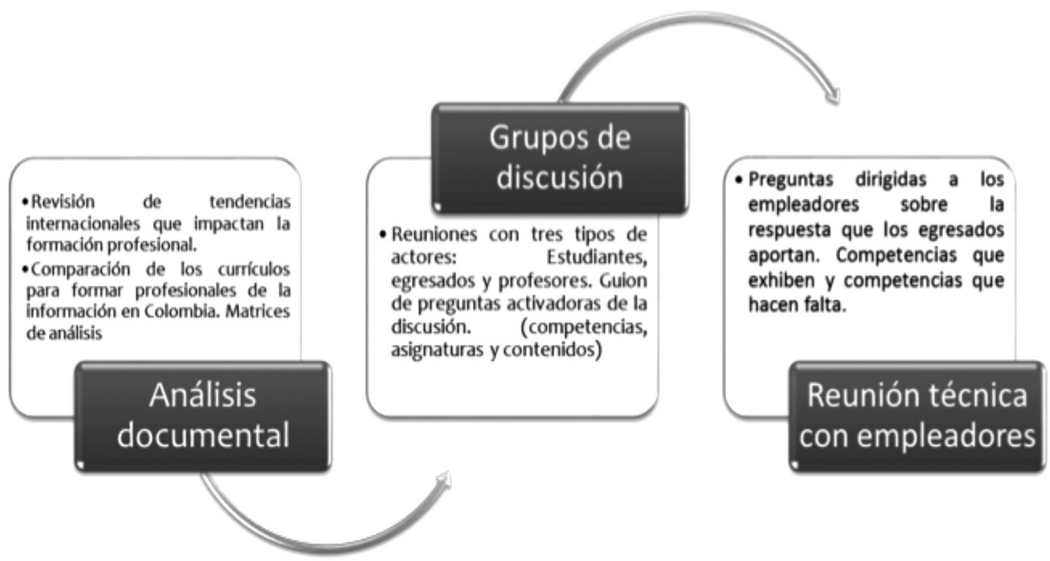

Figura 1. Recorrido metodológico de la redimensión curricular del Programa de Sistemas de Información y Documentación (PSID) Fuente: elaboración propia

\section{RESULTADOS: PRODUCTOS DE LA REDIMENSIÓN CURRICULAR}

Los productos medulares resultantes del proceso de actualización curricular son la actualización de la formación investigativa, la creación de una línea en sistemas de información, la realización de ajustes en la ubicación, denominación, contenidos o enfoques de algunos espacios académicos (asignaturas) y la inclusión de nuevas modalidades de grado. Estos elementos parten de la definición de los perfiles integral, general, institucional y profesional que orientan las competencias de acuerdo con los fundamentos curriculares del PSID. Otro producto resultado del proceso de actualización es la malla curricular, en la cual se evidencian los ejes, núcleos, áreas y espacios académicos, articulados con líneas de investigación identificadas por la Universidad de La Salle como prioritarias.

En cuanto a los perfiles integrales, el PSID define a la persona como el individuo educado integralmente con capacidad para la generación de conocimiento que aporte a la transformación social y productiva del país a través del desarrollo de sistemas, redes, productos y servicios de información en bibliotecas, centros de documentación y archivos que contribuyan a la democratización del acceso a la información.

En relación con el perfil general, el profesional en Sistemas de Información y Documentación es una persona íntegra que promueve el diálogo entre fe, ciencia y culturas, con sentido crítico, valores y sensibilidad social, capaz 
de promover, desarrollar, gestionar y liderar proyectos en unidades, redes y servicios de información bibliotecaria, documental y archivística que contribuyan a la construcción de nacionalidad y al desarrollo humano, económico, social y a la protección del medio ambiente.

El perfil institucional, en consonancia con el PEUL y el Estatuto Orgánico de la Universidad, plantea que el profesional en Sistemas de Información y Documentación proyecta y desarrolla su acción formativa inspirado en la tradición lasallista, siendo protagonista responsable de su propia formación, sensible a los contextos de exclusión, a las realidades de los jóvenes y a las urgencias educativas del momento, a través de una relación pedagógica fundada en el ejercicio responsable de la autonomía, la generación significativa de conocimiento y el compromiso decisivo con la transformación de la realidad, en armonía con los lineamientos trazados por el EFL.

El perfil profesional del egresado en Sistemas de Información y Documentación propone una persona que aprende porque investiga, capaz de promover y desarrollar, con la participación activa de la comunidad, sistemas de información bibliotecarios y archivísticos apoyados en las TIC que contribuyan al acceso democrático de la información y el conocimiento en términos de oportunidad, calidad y pertinencia, y al acceso y disfrute del patrimonio cultural documental, siempre en la constante búsqueda, conservación, transmisión y desarrollo del saber humano científico y tecnológico, con sentido universal, en beneficio del desarrollo del hombre integral, dentro de principios éticos y a partir de las características y condiciones de la sociedad, la cultura y los valores nacionales.

En relación con las competencias integrales y teniendo presentes los lineamientos curriculares institucionales y el análisis de la información recabada luego de haber aplicado las técnicas de investigación reseñadas en el punto referido al recorrido metodológico, el PSID definió las siguientes competencias que integran el ser, el saber y el hacer de la profesión desde los criterios expresados en el PEUL. Para ello, en primer lugar se realizó una definición a partir de la siguiente clasificación: competencias de generales, institucionales, y profesionales, asociadas con los criterios institucionales, las tendencias del mundo y la naturaleza del programa.

\section{Competencias generales}

a) Lidera proyectos pertinentes y de impacto social, inherentes a la práctica profesional, considerando las características del entorno político, económico, social y cultural, y promoviendo el acceso responsable a las decisiones y realizaciones de la comunidad. 
b) Trabaja en el desarrollo de proyectos multidisciplinares para dar solución a problemas socialmente sensibles con una visión integral del mundo, ejerciendo de manera responsable su propia autonomía.

c) Accede, usa y comunica información como medio para satisfacer las necesidades de formación que contribuyan a su desarrollo personal, laboral y profesional, durante toda la vida.

d) Participa en la formulación de políticas públicas de información que propendan por el desarrollo de la profesión, ejerciendo sus derechos ciudadanos con responsabilidad y compromiso social.

\section{Competencias institucionales}

a) Proyecta permanentemente nuevas tendencias locales, regionales y mundiales para el manejo de la información y el conocimiento, en armonía con los horizontes de sentido y con los fundamentos articuladores de la praxis universitaria formulada en el PEUL.

b) Genera cambio reconociendo el acceso a los sistemas y servicios de información como una oportunidad para el desarrollo institucional, la participación social, el respeto y defensa a la dignidad humana, y el desarrollo de proyectos para las clases menos favorecidas.

c) Reconoce en el trabajo corporativo y cooperativo disciplinar e interdisciplinar la estrategia articuladora para la democratización del acceso y uso de la información en función del desarrollo humano integral y sustentable.

\section{Competencias profesionales}

a) Gestiona, con pensamiento reflexivo y crítico, procesos en unidades, redes, sistemas y servicios de información, consultando los avances de la ciencia y las exigencias que plantea la sociedad de la información y el conocimiento.

b) Aplica con actitud creadora y decisiva, en la gestión de unidades y sistemas de información, conocimientos, técnicas y normas para la recolección, procesamiento analítico-sintético, almacenamiento, conservación, búsqueda y recuperación, diseminación y uso de los recursos de información, de acuerdo con las necesidades generales de la comunidad o el perfil específico del usuario.

c) Propone e implementa innovaciones para el desarrollo de unidades y servicios de información, adaptándose a los cambios del entorno con racionamiento crítico en el análisis y valoración de alternativas. 
d) Utiliza tecnologías de la información y la comunicación como medio para optimizar la circulación de los recursos de información a través de servicios y unidades de información, contribuyendo a la democratización del acceso a la ciencia, entendida como un componente central de la cultura, y al disfrute del patrimonio documental.

e) Evalúa y propone alternativas de mejora en los procesos de generación, tratamiento, transferencia y uso de la información y la actividad científica, conducentes a satisfacer con pertinencia y responsabilidad social las necesidades informativas de la comunidad.

f) Orienta a usuarios y productores de recursos y servicios de información bibliográfica y documental en su acceso y uso, observando la normatividad y las políticas adoptadas, buscando coadyuvar en el desarrollo integral de la persona, la transformación de la sociedad y el fomento de la cultura.

El primero de los resultados derivados de la actualización curricular fue el ajuste realizado a la formación investigativa (Tabla 1), en virtud de que en la malla curricular vigente se consideraba un espacio académico por semestre para lograr el aprendizaje y desarrollo de competencias investigativas, las cuales preparaban a los estudiantes para enfrentar con éxito la realización del trabajo de grado. Sin embargo, en la malla vigente, la denominación de estos espacios académicos se orientaba más hacia un producto o dimensión profesional específica (necesidades de información, organización documental y otros) que al abordaje de los diversos métodos, enfoques, tipos y técnicas de investigación: cuantitativa y cualitativa, particularizando las opciones metodológicas y técnicas del proceso de investigación científica.

Con este ajuste se logra organizar mejor la diacronía relacionada con los espacios académicos de formación investigativa, al darles espacio y tiempo curricular de una forma más lógica y coherente, discurriendo desde los paradigmas de investigación social hasta los métodos y técnicas de investigación aplicadas a los sistemas de información y documentación, bibliotecología y archivística.

El segundo de los aspectos fundamentales del proceso de actualización curricular fue la realización de ajustes en la ubicación, denominación, contenidos y enfoques de algunos espacios académicos (asignaturas) que integran las áreas de la formación en la Universidad de La Salle, a saber: Bibliotecología, Archivística, Tecnología, Administración, Lasallismo y las electivas.

En este sentido, el área de Bibliotecología tuvo cuatro asignaturas a las cuales se les modificó el enfoque, como información y referencia por servicios de referencia; usuarios, redes y sistemas de información por búsqueda y 
recuperación de la información; lenguajes documentales especializados por lenguajes documentales y gestión de información y conocimiento por gestión de información (Tabla 2).

\begin{tabular}{|c|c|c|c|}
\hline NÚCLEO & SEMESTRE & MALLAACTUAL & MALLA PROPUESTA \\
\hline \multirow[t]{3}{*}{1} & I & $\begin{array}{l}\text { Producción de conocimiento en } \\
\text { el sistema de información }\end{array}$ & $\begin{array}{l}\text { Paradigmas y enfoques de la } \\
\text { investigación social }\end{array}$ \\
\hline & $\|$ & $\begin{array}{l}\text { Sistema de información como } \\
\text { realidad social }\end{array}$ & $\begin{array}{l}\text { Métodos de investigación } \\
\text { cualitativa }\end{array}$ \\
\hline & III & $\begin{array}{l}\text { Necesidades de información de } \\
\text { los usuarios }\end{array}$ & $\begin{array}{l}\text { Métodos de investigación } \\
\text { cuantitativa }\end{array}$ \\
\hline \multirow[t]{3}{*}{2} & IV & $\begin{array}{l}\text { Organización de la información } \\
\text { documental }\end{array}$ & $\begin{array}{l}\text { Técnicas de investigación } \\
\text { documental }\end{array}$ \\
\hline & V & $\begin{array}{l}\text { Información relevante de } \\
\text { acuerdo a las necesidades }\end{array}$ & $\begin{array}{l}\text { Técnicas de recolección } \\
\text { y análisis de información } \\
\text { cualitativa }\end{array}$ \\
\hline & $\mathrm{VI}$ & $\begin{array}{l}\text { Análisis y sistematización de la } \\
\text { información documental }\end{array}$ & $\begin{array}{l}\text { Técnicas de recolección y } \\
\text { análisis de datos cuantitativos }\end{array}$ \\
\hline \multirow[t]{2}{*}{3} & VII & $\begin{array}{l}\text { Herramientas para la gestión } \\
\text { de información en la solución } \\
\text { de problemas }\end{array}$ & Proyecto de investigación \\
\hline & VIII & $\begin{array}{l}\text { Planes para la democratización } \\
\text { del conocimiento }\end{array}$ & $\begin{array}{l}\text { Diseño de metodología de } \\
\text { investigación }\end{array}$ \\
\hline \multirow[t]{2}{*}{4} & IX & Modalidad de Grado I & $\begin{array}{l}\text { Investigación aplicada al } \\
\text { proyecto de grado }\end{array}$ \\
\hline & $x$ & Modalidad de Grado II & Trabajo de grado \\
\hline
\end{tabular}

Tabla 1. Actualización de la formación investigativa del PSID

\begin{tabular}{|l|l|}
\hline \multicolumn{1}{|c|}{$\begin{array}{c}\text { PLAN DE ESTUDIOS ANTERIOR } \\
\text { (Asignaturas que se modifican) }\end{array}$} & \multicolumn{1}{c|}{$\begin{array}{c}\text { PLAN DE ESTUDIOS NUEVO } \\
\text { (Homologación) }\end{array}$} \\
\hline Fundamentos de la información & Fundamentos de la información \\
\hline Fuentes y recursos de información & Fuentes y recursos de información \\
\hline Información y referencia & Servicios de referencia \\
\hline Unidades, redes y sistemas de información & Búsqueda y recuperación de la información \\
\hline Estudios métricos de la información & Estudios métricos de la información \\
\hline Usuarios de información & Usuarios de información \\
\hline Introducción al análisis de información & Introducción al análisis de información \\
\hline Descripción bibliográfica & Descripción bibliográfica \\
\hline Análisis de información & Análisis de información \\
\hline Lenguajes documentales especializados & Lenguajes documentales \\
\hline
\end{tabular}




\begin{tabular}{|l|l|}
\hline Gestión de información y el conocimiento & Gestión de información \\
\hline Desarrollo de colecciones & Desarrollo de colecciones \\
\hline Lectura y lectores & Lectura y lectores \\
\hline Práctica profesional & Práctica profesional \\
\hline
\end{tabular}

Tabla 2. Cambios de enfoques de espacios académicos del área de Bibliotecología

El área de Archivística también fue objeto de cambios en cuanto a la denominación y enfoque de los espacios académicos que la conforman. En esencia, los cambios introducidos en esta área responden a la lógica de la apropiación del conocimiento en el proceso del ciclo de vida del documento. De modo que la asignatura Instituciones y documentos se modifica por Gestión documental I; Gestión documental por Gestión documental II; Derecho a la información por Derecho al acceso a la información y Conservación del patrimonio bibliográfico y documental por Preservación digital, con énfasis en los medios modernos (Tabla 3).

\begin{tabular}{|c|c|}
\hline $\begin{array}{c}\text { PLAN DE ESTUDIOS ANTERIOR } \\
\text { (Asignaturas que se modifican) }\end{array}$ & $\begin{array}{c}\text { PLAN DE ESTUDIOS NUEV0 } \\
\text { (Homologación) }\end{array}$ \\
\hline Fundamentos de archivística & Fundamentos de archivística \\
\hline Instituciones y documentos & Gestión documental I \\
\hline Gestión documental & Gestión documental II \\
\hline Clasificación y ordenación documental & Clasificación y ordenación documental \\
\hline Derecho a la información & Derecho de acceso a la información \\
\hline Descripción de archivos & Descripción de archivos \\
\hline Evaluación documental & Evaluación documental \\
\hline Conservación del patrimonio bibliográfico documental & Preservación de documento digital \\
\hline Diseño de programas de gestión documental & Diseño de programas de gestión documental \\
\hline Gestión de documentos electrónicos & Gestión de documentos electrónicos \\
\hline
\end{tabular}

Tabla 3. Cambios de enfoques de espacios académicos del área de Archivística

La línea de tecnología fue la que sufrió más cambios, en función de las necesidades de formación detectadas en los grupos de discusión y reuniones técnicas realizadas con empleadores. Las modificaciones de enfoques en esta área tienen que ver con la organización del proceso de diseño, estructuración, validación y pruebas de los sistemas de información. Conviene acotar que la perspectiva predominante en esta área fue que los profesionales de la información cuenten con el lenguaje y las herramientas metodológicas para dialogar con 
equipos de trabajo interdisciplinarios, con el compromiso de conceptualizar, diseñar e implantar sistemas de información en diversos escenarios y sectores productivos de la sociedad. De esta forma, el conocimiento y las prácticas bibliotecológicas y archivísticas se utilizan como los elementos medulares que dan impulso y cohesión a la construcción y diseño de los sistemas de información.

Dentro de los cambios más representativos en cuanto a los enfoques de asignaturas del área de tecnología se tienen los siguientes: la asignatura Fundamentos de las TIC se modificó por Introducción a los sistemas de información y Requerimientos de los sistemas de información; la asignatura Telemática y redes cambió el enfoque hacia Análisis de sistemas de información. En cuanto a la asignatura Análisis y diseño de sistemas, se modificó por Diseño de sistemas de información. Otra de las asignaturas del área de tecnología que se modificó fue Productos y estructuración de documentos digitales, la cual cambia su enfoque hacia otra asignatura con contenidos y competencias diferenciados, enfocados hacia la Construcción y evaluación de sistemas de información. También se modificó la asignatura Sistemas automatizados de gestión de información, dando paso a una nueva materia, denominada Implementación de sistemas de información. Finalmente, la asignatura electiva disciplinar se cambia por Soluciones a sistemas de información (Tabla 4).

\begin{tabular}{|c|c|}
\hline $\begin{array}{l}\text { PLAN DE ESTUDIOS ANTERIOR } \\
\text { (Asignaturas que se modifican) }\end{array}$ & $\begin{array}{l}\text { PLAN DE ESTUDIOS NUEVO } \\
\text { (Homologación) }\end{array}$ \\
\hline \multirow[t]{2}{*}{ Fundamentos de las TIC } & Introducción a los sistemas de información \\
\hline & Requerimientos en sistemas de información \\
\hline Telemática y redes & Análisis de los sistemas de información \\
\hline Análisis y diseño de sistemas & Diseño de sistemas de información \\
\hline $\begin{array}{l}\text { Productos y estructuración } \\
\text { de documentos digitales }\end{array}$ & $\begin{array}{l}\text { Construcción y evaluación de sistemas } \\
\text { de información }\end{array}$ \\
\hline Bases de datos documentales & Pruebas para los sistemas de información \\
\hline Sistemas automatizados de gestión de información & Implementación de sistemas de información \\
\hline Equivalente: electiva disciplinar & Soluciones a sistemas de información \\
\hline
\end{tabular}

Tabla 4. Cambios de enfoques de espacios académicos del área de Tecnología

Otra de las líneas curriculares que cambió, en cuanto a las denominaciones y orientaciones de sus contenidos, fue la de Administración. Las asignaturas que cambian fueron Gestión de calidad y Costos y presupuestos en unidades de información. Las asignaturas Servicios y productos de 
información y Mercadeo de la información se fusionan y pasan a ser Mercadeo de información; Formulación y evaluación de proyectos se reorienta por Gestión de proyectos. La relación de asignaturas que modificaron sus denominaciones y enfoques se presentan en la Tabla 5.

\begin{tabular}{|c|c|}
\hline $\begin{array}{c}\text { PLAN DE ESTUDIOS ANTERIOR } \\
\text { (Asignaturas que se modifican) }\end{array}$ & $\begin{array}{c}\text { PLAN DE ESTUDIOS NUEVO } \\
\text { (Homologación) }\end{array}$ \\
\hline Sociedad de la información y del conocimiento & Sociedad de la información y del conocimiento \\
\hline Administración & Administración \\
\hline Estadística & Probabilidad en unidades de información \\
\cline { 2 - 2 } & Muestreo en unidades de información \\
\hline Equivalente: electiva disciplinar & Gestión de calidad \\
\hline Equivalente: electiva disciplinar & Costos y presupuestos en unidades de información \\
\hline Gestión de talento humano & Gestión de talento humano \\
\hline Servicios y productos de información & Mercadeo de información \\
\hline Mercadeo de servicios de información & \\
\hline Planeación estratégica & Planeación estratégica \\
\hline Formulación y evaluación de proyectos & Gestión de proyectos \\
\hline Política social & Política social \\
\hline
\end{tabular}

Tabla 5. Cambios de enfoques de espacios académicos del área de Administración

Finalmente, el área de Lasallismo, que constituye la impronta de la universidad, mantiene la malla vigente: cátedra lasallista, Humanidades I, Humanidades II, Cultura religiosa I, II y III, Ética general y Ética en las profesiones. Las electivas tienen un giro que apoya la construcción de la interdisciplinariedad y el fortalecimiento de lo disciplinar puesto que le permite al estudiante acceder a formación de vanguardia frente a las demandas del mercado, de manera que la malla sigue siendo pertinente para la formación de los profesionales de la información ante las necesidades de los sectores de la economía y la industria.

También se mantienen de acuerdo a la estructura de la malla curricular actual tres electivas disciplinares, dos electivas que se ofrecen en otros programas de la Facultad de Ciencias Económicas y Sociales y una electiva interdisciplinaria. De esta forma se garantiza una formación profesional en sistemas de información y documentación, bibliotecología y archivística con un enfoque lasallista integral y consustanciado con las necesidades sociales. 
La malla curricular actualizada se estructura a partir de ejes, núcleos, áreas y espacios académicos, que se muestran articulados con líneas de investigación identificadas como prioritarias. En tal sentido, el tejido de relaciones de ejes curriculares, núcleos y áreas da como resultado la Malla Curricular del PSID. Se definen entonces como ejes estructurales del proceso curricular los siguientes: a) prácticas y problemas, que se alimenta en las necesidades del entorno, de la sociedad y del sector empresarial; b) ciencias y disciplinas, propio de las disciplinas, ciencias y saberes, y c) investigación, donde se dinamizan las competencias en la búsqueda de nuevo conocimiento.

Los ejes de prácticas y problemas están relacionados con:

a) Reconocimiento del papel de los sistemas bibliotecarios y archivísticos en la construcción de tejido social a partir de los procesos generados por la interacción información-documento-usuario-institución informativa documental.

b) Conocimientos y técnicas que intervienen en la identificación y organización de la información registrada en cualquier soporte como insumo para la participación en los beneficios de la Sociedad de la Información y el conocimiento.

c) Gestión de recursos, unidades, sistemas y servicios de información como medio para la generación de conocimiento y la innovación científica y tecnológica.

d) Democratización el acceso a la información con el fin de contribuir a aumentar las oportunidades para el desarrollo humano, económico, social y la protección del medio ambiente.

El eje de ciencias y disciplinas que se identificó como producto de la redimensión curricular fue:

a) Ciencia de la información, bibliotecología, documentación y archivistica: permitirá obtener los conocimientos necesarios para dinamizar las competencias relacionadas con el área específica de la profesión para la comprensión del sistema mediante el cual se interrelaciona información-documento-usuario-institución informativa documental.

b) Gestión y administración: saberes que dotarán a los estudiantes de los conocimientos y destrezas requeridos para la gestión y administración de los servicios, sistemas y redes de información y documentación, en 
el contexto de la institución informativa documental del sistema objeto de estudio del programa.

c) Informática: brindará al profesional las herramientas necesarias para aplicar las tecnologías de la información y las comunicaciones en los procesos de recuperación, organización, conservación y difusión de la información que circula dentro del sistema en el que interactúan información-documento-usuario-institución informativa documental.

d) Ciencias puras y aplicadas: aportará conocimientos relacionados con los principios y aplicaciones estadísticas necesarios para adelantar procesos investigativos que contribuyan al estudio del usuario objeto de las instituciones informativas documentales, así como de la producción documental y de conocimiento, y de desarrollo de la fundamentación de la profesión en todos sus aspectos.

e) Socioeconómicas: brindarán lo conocimientos necesarios para que el estudiante contextualice su accionar dentro de una praxis social que debe guiar el desarrollo profesional en función del desarrollo humano y sustentable.

f) Educación: conocimientos que le brindarán al profesional elementos para la dinamización de competencias relacionadas con la lectura y la escritura, como prácticas transversales en los procesos de difusión y uso de la información y el conocimiento.

Las líneas de investigación que se articularán al currículo del Programa de Sistemas de Información y Documentación, Bibliotecología y Archivística responden a los criterios rectores de la investigación en el pregrado, los lineamientos ya enunciados apuntan a la construcción de herramientas conceptuales, metodológicas y actitudinales que faciliten el desarrollo del espíritu de indagación en el futuro profesional (Tabla 6). Se debe promover el análisis de artículos de investigación, el desarrollo de habilidades para la búsqueda en bases de datos, el levantamiento de estados del arte y el conocimiento de los métodos más comunes de investigación según el campo de conocimiento. 


\begin{tabular}{|c|c|}
\hline \multicolumn{2}{|c|}{$\begin{array}{c}\text { Lista de investigaciones institucionales } \\
\text { Educación y Cultura } \\
\text { Memoria y Reconciliación } \\
\text { Territorio, Equidad y Desarrollo } \\
\text { Innovación y Tecnología } \\
\text { Ciudadanía, Ética y Política }\end{array}$} \\
\hline $\begin{array}{l}\text { Línea de Investigación de la Facultad de Ciencias } \\
\text { Económicas y Sociales y temáticas sobre las cuales } \\
\text { desarrollar la investigación }\end{array}$ & $\begin{array}{l}\text { Centros de investigación a los que se } \\
\text { encuentran asociados los programas } \\
\text { del área }\end{array}$ \\
\hline $\begin{array}{l}\text { Equidad y desarrollo } \\
\text { Temáticas de la línea cercanas al PSID: } \\
\text { - Desarrollo humano, integral y sustentable en } \\
\text { contextos locales, regionales y nacionales } \\
\text { - Derechos humanos y calidad de vida } \\
\text { - Políticas públicas en contexto de la equidad y desarrollo } \\
\text { - Desarrollo, información y sociedad } \\
\text { - Ciudadanía, inclusión y democracia }\end{array}$ & $\begin{array}{l}\text { Centros de Estudios en Desarrollo y Territorio } \\
\text { (CDET): generación de conocimiento sobre la } \\
\text { gestión y el desarrollo, el diseño de políticas } \\
\text { públicas, la democratización del conocimien- } \\
\text { to, el ejercicio democrático de la ciudadanía } \\
\text { capaz de asumir retos frente al desarrollo, que } \\
\text { aporten a la transformación social, cultural y } \\
\text { política. } \\
\text { Líneas de investigación: } \\
\text { 1. Políticas públicas, ciudadanía, democracia } \\
\text { y organizaciones } \\
\text { 2. Gestión, información, conocimiento } \\
\text { y desarrollo } \\
\text { 3. Alternatividad del desarrollo y } \\
\text { sustentabilidad }\end{array}$ \\
\hline \multicolumn{2}{|c|}{$\begin{array}{l}\text { Línea de Investigación del programa } \\
\text { Desarrollo, Cultura y Sociedad } \\
\text { Sublíneas de investigación } \\
\text { 1. Políticas públicas, agenciamiento cultural, desarrollo regional y local } \\
\text { 2. Lectura, escritura y desarrollo en la sociedad de la información } \\
\text { 3. Tecnologías de la información, del conocimiento e innovación } \\
\text { 4. Momoria, diálogo de saberes y participación ciudadana } \\
\text { 5. Estudios de información documental y enfoques de interpretación social }\end{array}$} \\
\hline $\begin{array}{l}\text { Grupo de Investigación del PSID } \\
\text { Desarrollo, Información y Sociedad }\end{array}$ & $\begin{array}{l}\text { Semilleros de Investigación del Programa } \\
\text { Aletheia } \\
\text { Arkeión } \\
\text { Amoxcali } \\
\text { Pharmakon }\end{array}$ \\
\hline
\end{tabular}

Tabla 6. Elementos estructurales de la investigación articulados al currículo del PSID

Fuente: Programa de Sistemas de Información y Documentación

Las temáticas de carácter disciplinar identificadas son las siguientes:

- El acceso a la información, abordado como un medio para mejorar la calidad de vida de la comunidad, garantizar la democratización del 
conocimiento y la equidad social así como la conformación del patrimonio documental que garantiza la construcción y recuperación de la memoria del país.

- La información como un derecho, en el sentido de entender la información en función de la sociedad, lo cual permite que los diferentes usuarios de la información documental puedan hacer valer sus derechos constitucionales (derecho de petición, acceso a la información, datos personales) y contar con los soportes necesarios para defender sus derechos fundamentales.

- La inclusión digital: las tecnologías de la información y la documentación han impactado toda la sociedad, pero llegar a la sociedad de la información implica más que contar con computadoras, significa hacer de las TIC herramientas de bienestar y desarrollo social. En respuesta a lo anterior, se avanza en la concepción de estrategias de e-inclusión o inclusión digital, entendida de acuerdo con el Observatorio de Sociedad, Gobierno y Tecnologías de Información como el ideal de acceso, uso y apropiación social de las tecnologías digitales para atender las necesidades de las comunidades, para promover las políticas públicas, la creación de conocimientos, la elaboración de contenidos apropiados y el fortalecimiento de la capacidad de las personas.

- Desarrollo de habilidades informativas: los profesionales de la información son los indicados para trabajar en el desarrollo de competencias informativas que le permitan a cualquier persona recuperar y utilizar la información no sólo para su vida diaria sino también para sus necesidades académicas; esto implica, de acuerdo con las Directrices IFLA, desde una alfabetización básica (leer y escribir) hasta la alfabetización informacional (uso de las tecnologías de la información y la comunicación).

- Lectura: la promoción de la lectura es la herramienta más claramente identificada como medio para la inclusión social. Este hecho se ve reflejado en la XIII Cumbre Iberoamericana de Jefes de Estado y de Gobierno, en donde se firmó la Declaración de Santa Cruz de la Sierra (2003) y se aprobó que el Plan Iberoamericano de Lectura Ilimita sea un programa cumbre, reconociendo que la lectura es un instrumento real para la inclusión social y un factor básico para el desarrollo social, cultural y económico de nuestros países. Esto evidencia la necesidad de fortalecer los programas de lectura no sólo en el aula de clase, sino dentro de los programas de extensión universitaria.

- La formación de usuarios: más allá de la enseñanza del uso de la biblioteca, de los servicios, etc., el bibliotecólogo puede aprovechar este espacio para formar ciudadanos, tal como se indica dentro del 
programa de competencias ciudadanas del Ministerio de Educación Nacional, es decir, resulta imprescindible formar personas sensibles, críticas, respetuosas, justas, libres y autónomas con sentido de pertenencia a una comunidad, capaces de solucionar los conflictos por vías pacíficas para construir efectivamente una sociedad participativa y democrática.

- Los archivos como centros de memoria: todo pueblo que no conoce su historia está destinado a repetirla; en tal sentido, conocer la funcionalidad, operatividad y forma de acceder a la información presencial o virtual permite conocer quiénes somos, dónde estamos y a dónde queremos llegar. En otras palabras, los archivos como centros de memoria permiten la inclusión social al garantizar los derechos de todos y cada uno de los ciudadanos y la defensa de sus intereses.

- La necesidad de información y su correspondiente búsqueda son procesos inherentes al ser mismo de las personas, y exigen un continuo proceso de perfeccionamiento y autoconocimiento del espíritu humano y su relación con el entorno, por lo tanto, transforman continuamente la realidad. El programa ve en la necesidad de información documental la oportunidad de analizar, construir y aportar desde una sintaxis lógica el manejo de los documentos, los cuales son estudiados como un producto social y cultural, que constituye el resultado de la objetivación y del pensamiento y tienen como función conservar la memoria social.

- Control social: los archivistas como custodios del patrimonio documental en las organizaciones y en el país son los llamados a generar las políticas de conservación y difusión necesarias y suficientes, así como a garantizar su aplicación para que las organizaciones cuenten con los elementos de juicio que permitan demostrar sus acciones en el tiempo y frente a terceros, así como proporcionar a la comunidad la información válida que le permita ejercer el control social que todo ciudadano debe ejercer sobre sus instituciones.

Los espacios académicos se distribuyen considerando las áreas curriculares con sus pesos porcentuales, según los criterios y parámetros establecidos por la Universidad de La Salle en lo que respecta a diseño y estructuración de sus currículos. La Tabla 7 presenta la relación entre los espacios académicos, las áreas curriculares, los núcleos y los semestres, incluyendo además los porcentajes pautados para cada área curricular. 


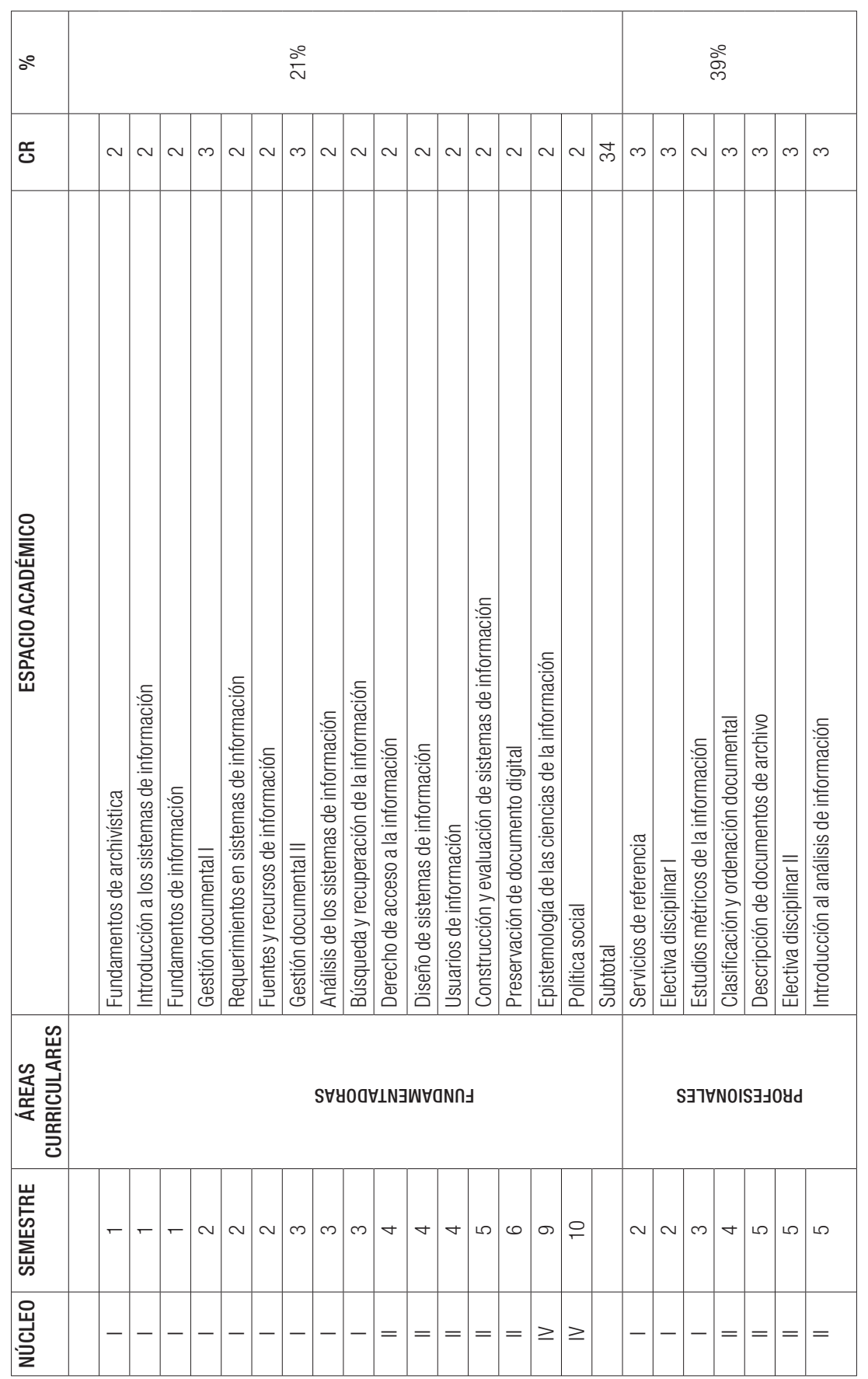



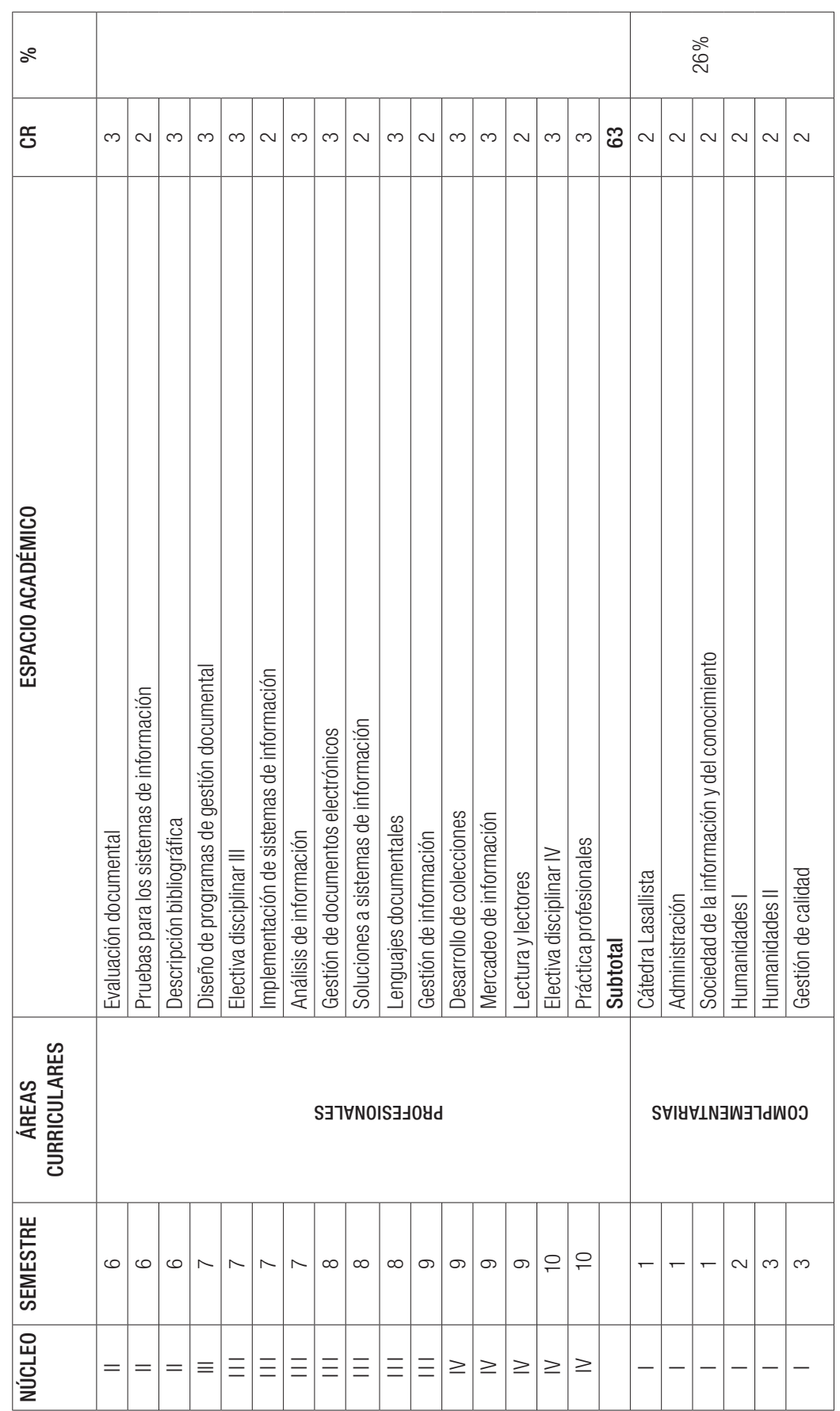


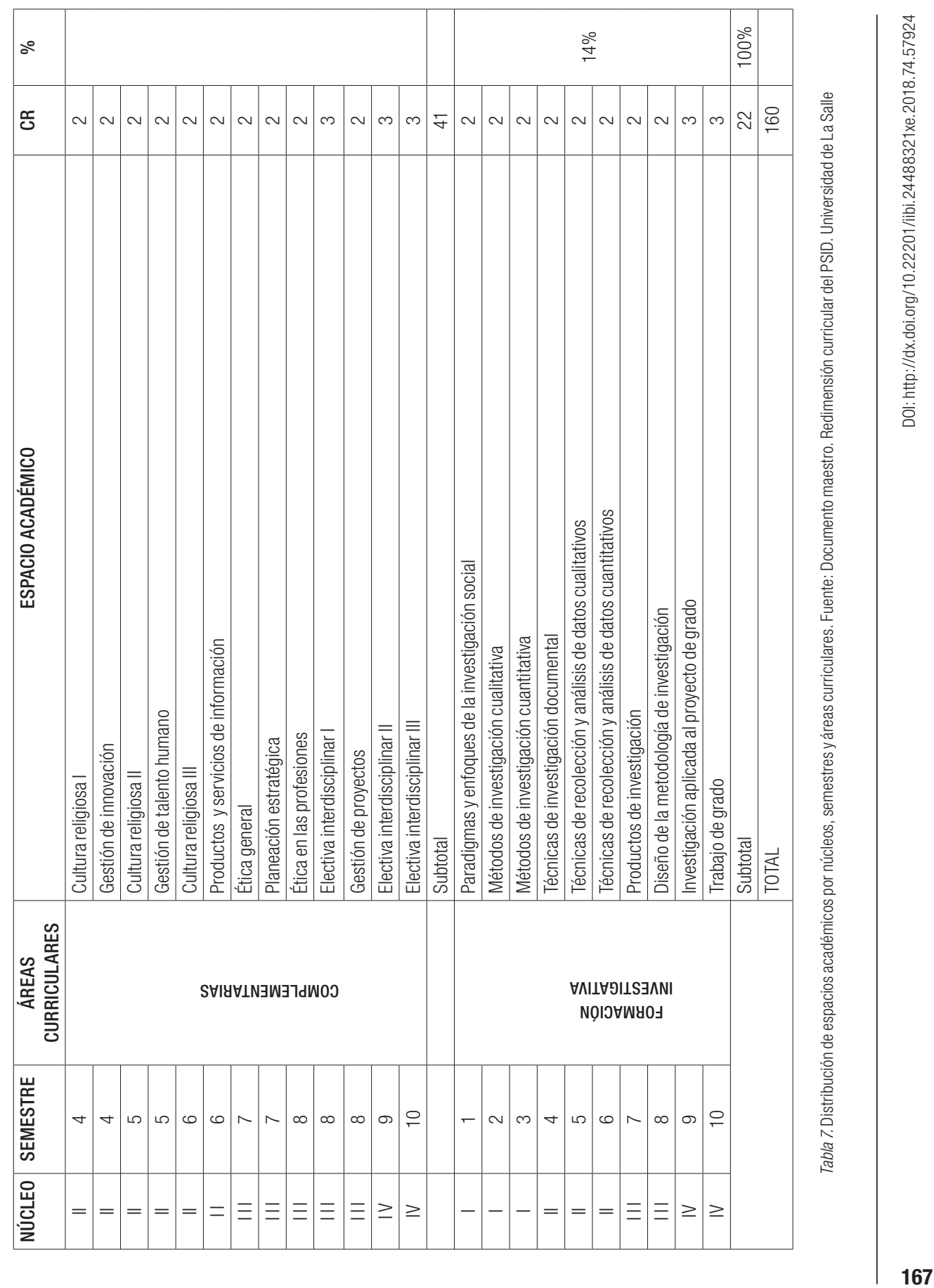




\section{Conclusiones}

El proceso de actualización curricular realizado en el Programa de Sistemas de Información y Documentación (PSID) de la Universidad de La Salle se asumió como un proyecto de investigación institucional, que estuvo orientado a lograr una mayor articulación de la malla con las necesidades y tendencias de la disciplina y de la profesión bibliotecológica y archivística, las demandas de los mercados laborales y sobre la base del Proyecto Educativo y Enfoque Formativo Lasallistas, que apelan a la formación de personas-profesionales con elevada sensibilidad social y conscientes de que su acción tiene una incidencia significativa en el desarrollo de procesos de desarrollo humano integral sostenible, apoyados en el acceso y uso estratégico de la información.

La actualización que se deriva del proceso de investigación se concretó en la realización de importantes ajustes a la malla curricular, para alinearla más al perfil de los profesionales que se requieren en los sistemas de información y documentación del contexto colombiano, pero con visión global, lo cual es un elemento evidenciado en la consulta con empleadores, estudiantes de los últimos semestres, egresados y profesores del programa. De modo pues que la actualización puso su acento en el fortalecimiento y orden lógico de las líneas curriculares sustantivas para la formación de bibliotecólogos y archivistas, tales como bibliotecología, archivística, sistemas de información, gestión e investigación formativa, la cual debe establecer un continuo en la formación que discurra desde el pregrado hasta la formación de posgrado en el área.

\section{REFERENCIAS}

IFLA. 2014. Lyon Declaration on Access to Information and Development launched. Fecha de consulta: 24 de junio de 2015. http://conference.ifla.org/past-wlic/2014/ ifla80/node/522.html

IFLA. 2013. Surcando las olas o atrapados en la marea. Navegando en el entorno en evolución de la información, Percepciones del IFLA Trend Report. Fecha de consulta: 4 de febrero de 2014. http://www.abinia.org/surcando_las_olas_o_atrapados_en_la_marea.pdf

Inciarte, A., A. J. Bozo y M. C. Parra. 2012. "Reconceptualización de la calidad universitaria: un reto para América Latina”. Avaliação Campinas 17 (3) (noviembre): 637-660. Fecha de consulta: 2 de mayo de 2016. http://www.scielo.br/pdf/aval/ v17n3/a05v17n3.pdf 
Pirela, J. 2015. "La formación del profesional de la información frente al desafío de las sociedades hiperconectadas y los nuevos grupos”, en Información, entorno y evolución: visiones académicas y profesionales sobre el Informe de Tendencias de la IFLA, Jaime Ríos Ortega (coord.), 223-236. México: UNAM, Instituto de Investigaciones Bibliotecológicas y de la Información: IFLA, Oficina Regional para América Latina y el Caribe. Fecha de consulta: 24 de julio de 2015. http://iibi. unam.mx/publicaciones/293/informacion_entorno_evolucion_informe_tendencias_ifla\%20La\%20formacion\%20profesional\%20Johan\%20Pirela.html

Unesco. 2009. Conferencia Mundial sobre la Educación Superior. La nueva dinámica de la educación superior y la investigación para el cambio social y el desarrollo. Fecha de consulta: 2 de mayo de 2016. http://www.unesco.org/education/WCHE2009/comunicado_es.pdf

Universidad de La Salle. 2007. Proyecto Educativo Universitario Lasallista (PEUL). Bogotá, Colombia. Fecha de consulta: 25 de julio de 2015. https://www.lasalle. edu.co/wcm/myconnect/01d5946c-2fb2-49bf-b731-19b5f9180b53/proyecto-educativo-universitario-lasallista-peul.pdf?MOD=AJPERES \&CVID=1Wr3EqK\&CVI$\mathrm{D}=1 \mathrm{~W} r 3 \mathrm{EqK} \& \mathrm{CVID}=1 \mathrm{Wr} 3 \mathrm{EqK} \& \mathrm{CVID}=1 \mathrm{~W} \mathrm{r} 3 \mathrm{EqK}$

Universidad de La Salle. 2008a. Enfoque Formativo Lasallista (EFL). Bogotá, Colombia. Fecha de consulta: 24 de julio de 2015. http://www.lasalle.edu.co/wps/wcm/ connect/e2b72d85-7970-4361-a8b2-c8026ce6b53c/28-enfoque-formativo-lasallista-efl.pdf?MOD=AJPERES

Universidad de La Salle. 2008b. Lineamientos para la redimensión permanente de la malla curricular. Fecha de consulta: 24 de julio de 2015. Bogotá, Colombia. http:/www.lasalle.edu.co/wps/wcm/connect/b278afbe-8f00-47f2-bc4d-85dab3163d0a/30-lineamientos-para-la-redimensi\%C3\%B3n-permanente-de-la-malla-curricular.pdf?MOD=AJPERES

Para citar este texto:

Pirela Murillo, Johann y Nelson Javier Pulido Daza. 2018. “Actualización curricular del Programa de Sistemas de Información y Documentación de la Universidad de La Salle-Colombia”. Investigación Bibliotecológica: archivonomía, bibliotecología e información 32 (74): 145-169. http://dx.doi.org/10.22 201/iibi.24488321xe.2018.74.57924 